\title{
Measuring Dabigatran Levels: What Tests Are Available and What Still Needs to Be Done?
}

\author{
Noel C. Chan, M.B.B.S. ${ }^{1,2}$ Jeffrey I. Weitz, M.D. ${ }^{1,2,3}$
}

${ }^{1}$ Thrombosis and Atherosclerosis Research Institute, Hamilton, Canada

2 Department of Medicine, McMaster University, Hamilton, Canada

3 Department of Biochemistry and Biomedical Sciences, McMaster

University, Hamilton, Canada

Thromb Haemost 2017;117:2213-2214.

Dabigatran is an oral thrombin inhibitor that is licensed for stroke prevention in atrial fibrillation and for prevention and treatment of venous thromboembolism. Although dabigatran is administered in fixed doses without routine coagulation monitoring, quantification of the plasma levels of dabigatran is useful to detect accumulation in patients with acute kidney injury, for assessment of its potential contribution to serious bleeding, for determination of the optimal timing of urgent surgery and for identification of patients with acute ischemic stroke who can safely receive fibrinolytic therapy. ${ }^{1,2}$

With the introduction of idarucizumab, the availability of assays that can quantify plasma dabigatran concentrations is essential to identify appropriate candidates for reversal and to monitor them to ensure that reversal is achieved. This reflects the use of this reversal agent in serious bleeding or where patients on dabigatran require urgent surgery or interventional procedures., ${ }^{3,4}$ Routine coagulation assays such as the activated partial thromboplastin time (aPTT) and thrombin time are responsive to the anticoagulant effects of dabigatran. However, the aPTT lacks sensitivity and can be normal in the face of significant amounts of dabigatran, whereas the thrombin time is so sensitive that it is prolonged even by low concentrations of dabigatran. Therefore, there is an urgent need for widespread availability of rapid and accurate assays to quantify dabigatran levels.

Both chromogenic and clot-based assays to quantify dabigatran levels are commercially available (-Table $\mathbf{1}$ ). The tests use thrombin (IIa) or ecarin to initiate clotting and they have been validated by comparing the results with dabigatran concentrations measured by liquid chromatography tandem mass spectroscopy (LC-MS/MS), the gold standard for dabigatran quantification. Both types of assays can be performed on commercially available coagulometers with rapid turnaround.
Address for correspondence Jeffrey I. Weitz, M.D., Thrombosis and Atherosclerosis Research Institute, 237 Barton Street East, Hamilton, ON L8L 2X2, Canada (e-mail: weitzj@taari.ca).

In this issue of Thrombosis and Haemostasis, Poli et al used 288 stored samples from 48 dabigatran-treated patients to compare the diagnostic accuracy of the Biophen direct thrombin inhibitor (BDTI) assay, a new chromogenic anti-Ila assay, and the currently available clot-based Hemoclot thrombin inhibitor (HTI) assay, both of which are manufactured by Hyphen BioMed (Neuville-sur-Oise, France). The results with the two assays were compared with dabigatran concentrations measured using LC-MS/MS. After establishing round-the-clock availability of the BDTI assay, its test performance in routine clinical practice was then further evaluated in 150 fresh citrated blood samples collected from 25 dabigatran-treated patients.

The BDTI assay has several potential advantages over the HTI assay. First, compared with LC-MS/MS, there is closer agreement with the BDTI assay than with the HTI assay. Second, with dabigatran concentrations below $60 \mathrm{ng} / \mathrm{mL}$, the BDTI assay appears to be more accurate than the HTI assay because fewer samples are misclassified. Thus, in several of the tested samples, dabigatran was detected with the HTI assay when none was detectable by LC-MS/MS. Although the BDTI assay did not suffer from this deficiency, it reported absence of dabigatran in a few samples where levels as high as $40 \mathrm{ng} / \mathrm{mL}$ were detected by LC-MS/MS. Therefore, neither test is perfect. Third, once reconstituted, reagents for the BDTI assay have longer stability at room temperature than those for the HTI assay (72 and 8 hours, respectively), which may be advantageous for busy clinical laboratories. Finally, the BDTI assay is less sensitive to heparin than the HTI assay, although neither assay detects heparin at levels below $1 \mathrm{IU} / \mathrm{mL}$, which covers the usual therapeutic range.

Does the enhanced accuracy of the BDTI assay over the HTI assay really matter from a clinical perspective? Accuracy is important particularly with low levels of dabigatran. Thus, misclassification of a level as being low could lead to bleeding if patients undergo major surgery or if thrombolysis is given for 
Table 1 Commercially available assays for quantifying dabigatran concentrations

\begin{tabular}{|l|l|l|}
\hline Method & Principle & Specific assays \\
\hline Chromogenic & Thrombin-based & $\begin{array}{l}\text { Hyphen Biomed } \\
\text { Biophen DTI }\end{array}$ \\
\cline { 2 - 3 } & Ecarin-based & Stago ECA-II \\
\hline Clot-based & Thrombin-based & Hyphen Biomed HTI \\
\cline { 3 - 3 } & & HemosIL DTI \\
\cline { 3 - 3 } & & Technoclot DTI \\
\cline { 3 - 3 } & & Roche Dilute \\
& & Thrombin Time \\
\cline { 3 - 3 } & & Siemens INNOVANCE \\
& & DTI \\
\hline
\end{tabular}

Abbreviations: DTI, Direct thrombin inhibitor; ECA, ecarin chromogenic assay; HTI, Hemoclot thrombin inhibitor.

Source: Adapted from European Medicines Agency. ${ }^{8}$

treatment of acute ischemic stroke. In reality, however, with the widespread availability of idarucizumab, clinicians often reverse dabigatran before waiting for the results of coagulation assays in life-threatening or emergency circumstances. ${ }^{5,6}$ Therefore, despite the advantages of the BDTI assay over the HTI assay, the more important factor is ensuring widespread availability of at least one of these tests.

Access to quantitative assays for dabigatran remains problematic. Despite the availability of numerous assays ( - Table 1), none are approved in the United States. In Ontario, the most populous province in Canada, only $4 \%$ of clinical laboratories offer these assays. Likewise, based on data from the Royal College of Pathologists of Australasia Quality Assurance Program and a survey by the United Kingdom National External Quality Assessment Service, approximately 26\% of laboratories in Australasia and $12 \%$ of those in the United Kingdom offer quantitative assays for dabigatran. ${ }^{7,8}$ Barriers to the uptake and implementation of quantitative assays include the cost of implementing new assays in an environment of shrinking hospital budgets and the current lack of regulatory body-approved reagents, calibrators and methods in some jurisdictions. Therefore, much work still needs to be done.

In conclusion, the BDTI assay represents another step forward in the development of quantitative assays for dabigatran. We now have the means to rapidly and accurately measure dabigatran levels. However, implementation of these assays at the bedside requires cooperation among regulators, standardization committees and hospitals. Such cooperation is urgently needed.

Conflicts of interest

J. Weitz and N. Chan received research funding from Diagnostica Stago for evaluation of the ECA-II assay.

\section{References}

1 Garcia D, Barrett YC, Ramacciotti E, Weitz JI. Laboratory assessment of the anticoagulant effects of the next generation of oral anticoagulants. J Thromb Haemost 2013;11(02):245-252

2 Ageno W, Büller HR, Falanga A, et al. Managing reversal of direct oral anticoagulants in emergency situations. Anticoagulation Education Task Force White Paper. Thromb Haemost 2016;116(06):1003-1010

3 Pollack CV Jr, Reilly PA, van Ryn J, et al. Idarucizumab for dabigatran reversal - full cohort analysis. N Engl J Med 2017;377(05):431-441

4 Pollack CV Jr, Reilly PA, Bernstein R, et al. Design and rationale for RE-VERSE AD: a phase 3 study of idarucizumab, a specific reversal agent for dabigatran. Thromb Haemost 2015;114(01):198-205

5 Levy JH, Ageno W, Chan NC, Crowther M, Verhamme P, Weitz JI; Subcommittee on Control of Anticoagulation. When and how to use antidotes for the reversal of direct oral anticoagulants: guidance from the SSC of the ISTH. J Thromb Haemost 2016;14(03):623-627

6 Greinacher A, Thiele T, Selleng K. Reversal of anticoagulants: an overview of current developments. Thromb Haemost 2015;113(05): 931-942

7 Favaloro EJ, Bonar R, Butler J, Marsden K. Laboratory testing for the new oral anticoagulants: a review of current practice. Pathology 2013;45(04):435-437

8 European Medicines Agency. The role of pharmacokinetic and pharmacodynamic measurements in the use of direct oral anticoagulants: Measuring the concentration of the direct thrombin inhibitor (dabigatran etexilate). Available at http://www.ema. europa.eu/docs/en_GB/document_library/Presentation/2016/01/ WC500199525.pdf. Accessed 22 September 22, 2017 